\title{
Space-Time Description of Scalar Particle Creation by a Homogeneous Isotropic Gravitational Field
}

\author{
Yu. V. Pavlov* \\ A. Friedmann Laboratory for Theoretical Physics, St. Petersburg, Russia, and \\ Institute of Mechanical Engineering, Russian Acad. Sci., 61 Bolshoy pr., St. Petersburg 199178, Russia
}

\begin{abstract}
We give the generalization of the method of the space-time description of particle creation by a gravitational field for a scalar field with nonconformal coupling to the curvature. The space-time correlation function is obtained for a created pair of the quasi-particles, corresponding to a diagonal form of the instantaneous Hamiltonian. The case of an adiabatic change of the metric of homogeneous isotropic space is analyzed. We show that the created pairs of quasi-particles in de Sitter space should be interpreted as pairs of virtual particles.
\end{abstract}

PACS number: 04.62.+v, 03.70.+k

\section{INTRODUCTION}

Quantum field theory in curved space-time is nowadays a sufficiently deeply elaborated area of theoretical physics (see the monographs [1, 2]) with important applications in cosmology and astrophysics. In particular, creation of particles with GUT-scale masses by the gravitational field of the early Universe may be used for an explanation of the observed visible and dark matter density 3 .

In the description of particle creation by the gravitational field, a widely used method is that of instantaneous Hamiltonian diagonalization [1] suggested by A. A. Grib and S. G. Mamayev [4]. A detailed study of the created pair formation process was performed in [5] for the case of a scalar field conformally coupled to the curvature. The method of a spacetime correlation function suggested in [5] made it possible to distinguish real created particles from virtual ones, to reveal the role of horizons in particle creation etc.

In quantum field theory in curved space-time, one frequently considers scalar field nonconformally coupled to gravity, in particular, the minimally coupled one. In such cases, the contributions related to nonconformal coupling may be dominant both in the particle creation effect [6] and in the vacuum averages values of the stress-energy tensor (see, e.g., [7]). Such a coupling with the curvature also takes place in the case of massive vector mesons (the longitudinal components [1). Conformal invariance is lacking

\footnotetext{
${ }^{*}$ E-mail: yuri.pavlov@mail.ru
}

in the graviton equations as well [8]. Therefore, it appears to be necessary to generalize the method of studying the particle creation process suggested in [5] to the nonconformal case. Moreover, some authors [9] consider the absence of such a generalization as an argument in favor of choosing only the conformal coupling in the wave equation for a scalar field.

The present paper suggests a generalization of the space-time description of particle creation [5] to the case of nonconformal coupling. In Section 2 we perform quantization of a general-type nonconformally coupled scalar field in homogeneous isotropic space. In Section 3, we carry out diagonalization of the generalized Hamiltonian built in [10, which, in the nonconformal case, allows one to solve the wellknown problem of an infinite density of quasiparticles created [11. In Section 4, we build a space-time correlation function for quasiparticles corresponding to the diagonal form of the instantaneous Hamiltonian and study the case of an adiabatically changing metric. In Section 5, we consider particle creation and the space-time correlation function in de Sitter space. The conclusion briefly sums up the results of the paper.

We use the system of units in which $\hbar=c=1$. The signs of the curvature tensor and the Ricci tensor are chosen in such a way that $R_{i k}=R_{i l k}^{l}$,

$$
R_{j k l}^{i}=\partial_{l} \Gamma_{j k}^{i}-\partial_{k} \Gamma_{j l}^{i}+\Gamma_{n l}^{i} \Gamma_{j k}^{n}-\Gamma_{n k}^{i} \Gamma_{j l}^{n},
$$

where $\Gamma_{j k}^{i}$ are the Christoffel symbols. 


\section{SCALAR FIELD IN CURVED SPACE}

Consider a complex scalar field $\varphi(x)$ with mass $m$, the Lagrangian

$$
L(x)=\sqrt{|g|}\left[g^{i k} \partial_{i} \varphi^{*} \partial_{k} \varphi-\left(m^{2}+V_{g}\right) \varphi^{*} \varphi\right],
$$

and the corresponding equation of motion

$$
\left(\nabla^{i} \nabla_{i}+V_{g}+m^{2}\right) \varphi(x)=0,
$$

where $\nabla_{i}$ are covariant derivatives in $N$-dimensional space-time with the metric $g_{i k}, g=\operatorname{det}\left(g_{i k}\right), \quad V_{g}$ is a function of invariant combinations of the metric tensor $g_{i k}$ and its partial derivatives.

Eq. (2) is conformally invariant if $m=0$ and $V_{g}=\xi_{c} R$, where $R$ is the scalar curvature and $\xi_{c}=(N-2) /[4(N-1)]$ (conformal coupling). The case $V_{g}=0$ corresponds to minimal coupling. An arbitrary $V_{g}$ leads to the advent of third- and higherorder derivatives of the metric in the metric stressenergy tensor of the scalar field and consequently in the Einstein equations.

It is well known that additional terms with higherorder derivatives appearing in equations lead to radical changes in the theory even if the coefficients of these terms are small.

If one requires that the metric stress-energy tensor should not contain derivatives of the metric of orders higher than two, then the following function is admissible as $V_{g}$ :

$$
V_{g}=\xi R+\zeta R_{G B}^{2},
$$

where

$$
R_{G B}^{2} \stackrel{\text { def }}{=} R_{l m p q} R^{l m p q}-4 R_{l m} R^{l m}+R^{2}
$$

(the Gauss-Bonnet coupling [12]).

Let us note that for $N=4$, with constant $\varphi(x)$, the contribution to the metric stress-energy tensor from the term with $R_{G B}^{2}$ is absent because the corresponding variation derivative vanishes [13. But for a variable $\varphi(x)$, a contribution from such terms could be taken into account if the constant $\zeta$ with the dimension (mass) ${ }^{-2}$ is nonzero.

Accounting for a possible coupling between a scalar field and the Gauss-Bonnet invariant $R_{G B}^{2}$ may play an important role in the early Universe; effects from a nonzero value of the parameter $\zeta$ in scalar field equations may appear in black-hole radiation, may affect the parameters of the so-called boson stars etc. The question of the values of the parameters $\zeta$ and $\xi$ are ultimately related to the area of the experiment.
Furthermore, without specifying $V_{g}$, let us consider an $N$-dimensional homogeneous isotropic space-time, choosing the metric in the form

$$
d s^{2}=g_{i k} d x^{i} d x^{k}=a^{2}(\eta)\left(d \eta^{2}-d l^{2}\right),
$$

where $d l^{2}=\gamma_{\alpha \beta} d x^{\alpha} d x^{\beta}$ is the metric of an $(N-1)$ dimensional space of constant curvature $K=0, \pm 1$.

The complete set of solutions to Eq. (2) in the metric (5) may be found in the form

$$
\varphi(x)=\frac{\tilde{\varphi}(x)}{a^{(N-2) / 2}(\eta)}=a^{-(N-2) / 2}(\eta) g_{\lambda}(\eta) \Phi_{J}(\mathbf{x}),
$$

where

$$
\begin{gathered}
g_{\lambda}^{\prime \prime}(\eta)+\Omega^{2}(\eta) g_{\lambda}(\eta)=0 \\
\Omega^{2}(\eta)=\left(m^{2}+V_{g}-\xi_{c} R\right) a^{2}+\lambda^{2} \\
\Delta_{N-1} \Phi_{J}(\mathbf{x})=-\left(\lambda^{2}-\left(\frac{N-2}{2}\right)^{2} K\right) \Phi_{J}(\mathbf{x})
\end{gathered}
$$

the prime denotes a derivative with respect to the conformal time $\eta$, and $J$ is the set of indices (quantum numbers) numbering the eigenfunctions of the Laplace-Beltrami operator $\Delta_{N-1}$ in $(N-1)$ dimensional space.

According to the Hamiltonian diagonalization method 1] (see the case of an arbitrary function $V_{g}$ in [14), the functions $g_{\lambda}(\eta)$ should obey the following initial conditions:

$$
g_{\lambda}^{\prime}\left(\eta_{0}\right)=i \Omega\left(\eta_{0}\right) g_{\lambda}\left(\eta_{0}\right), \quad\left|g_{\lambda}\left(\eta_{0}\right)\right|=\Omega^{-1 / 2}\left(\eta_{0}\right) .
$$

To perform quantization, let us expand the field $\varphi(x)$ in the complete set of solutions (6)

$$
\varphi(x)=\int d \mu(J)\left[\varphi_{J}^{(+)} a_{J}^{(+)}+\varphi_{J}^{(-)} a_{J}^{(-)}\right],
$$

where $d \mu(J)$ is a measure on the set of quantum numbers,

$$
\varphi_{J}^{(+)}(x)=\frac{g_{\lambda}(\eta) \Phi_{J}^{*}(\mathbf{x})}{\sqrt{2} a^{(N-2) / 2}(\eta)}, \quad \varphi_{J}^{(-)}(x)=\left(\varphi_{J}^{(+)}(x)\right)^{*},
$$

and require that the standard commutation relations hold for $a_{J}^{( \pm)}$and $\stackrel{*}{a}( \pm)$.

Let us build the Hamiltonian as the canonical one for the variables $\tilde{\varphi}(x)$ and $\tilde{\varphi}^{*}(x)$, for which the equation of motion does not contain their first-order derivatives with respect to the time $\eta$ [14. Recall that the equations of motion do not change after adding a full divergence $\partial J^{i} / \partial x^{i}$ to the Lagrangian density $L(x)$. 
Let us choose, in the coordinate system $(\eta, \mathbf{x})$, the vector

$$
\left(J^{i}\right)=\left(\sqrt{\gamma} c \tilde{\varphi}^{*} \tilde{\varphi}(N-2) / 2,0, \ldots, 0\right),
$$

where $\gamma=\operatorname{det}\left(\gamma_{\alpha \beta}\right), c=a^{\prime} / a$. Then, using the Lagrangian density $L^{\Delta}(x)=L(x)+\partial J^{i} / \partial x^{i}$, we obtain for the momenta canonically conjugate to $\tilde{\varphi}$ and $\tilde{\varphi}^{*}$ :

$$
\pi \equiv \frac{\partial L^{\Delta}}{\partial \tilde{\varphi}^{\prime}}=\sqrt{\gamma} \tilde{\varphi}^{* \prime}, \quad \pi_{*} \equiv \frac{\partial L^{\Delta}}{\partial \tilde{\varphi}^{* \prime}}=\sqrt{\gamma} \tilde{\varphi}^{\prime},
$$

respectively. Integrating the Hamiltonian density $h(x)=\tilde{\varphi}^{\prime} \pi+\tilde{\varphi}^{* \prime} \pi_{*}-L^{\Delta}(x)$ over the hypersurface $\Sigma: \eta=$ const, we obtain the following expression for the canonical Hamiltonian:

$$
\begin{aligned}
& H(\eta)=\int_{\Sigma} d^{N-1} x \sqrt{\gamma}\left\{\tilde{\varphi}^{* \prime} \tilde{\varphi}^{\prime}+\gamma^{\alpha \beta} \partial_{\alpha} \tilde{\varphi}^{*} \partial_{\beta} \tilde{\varphi}+\right. \\
& \left.\left[\left(m^{2}+V_{g}\right) a^{2}-\frac{N-2}{4}\left(2 c^{\prime}+(N-2) c^{2}\right)\right] \tilde{\varphi}^{*} \tilde{\varphi}\right\}
\end{aligned}
$$

(see a justification of such a choice of the Hamiltonian in 10, 14, and in Section 3).

The Hamiltonian (14) may be written in terms of the operators $a_{J}^{( \pm)}$and $\stackrel{*}{a}_{J}^{( \pm)}$in the following way:

$$
\begin{aligned}
H(\eta) & =\int d \mu(J)\left[E_{J}(\eta)\left(\stackrel{*}{a_{J}^{(+)}} a_{J}^{(-)}+\stackrel{*}{a}_{\bar{J}}^{(-)} a_{\bar{J}}^{(+)}\right)+\right. \\
& \left.+F_{J}(\eta) \stackrel{*}{a}(+) a_{\bar{J}}^{(+)}+F_{J}^{*}(\eta) \stackrel{*(-)}{(-)} a_{J}^{(-)}\right], \quad(15)
\end{aligned}
$$

where

$$
E_{J}=\frac{\left|g_{\lambda}^{\prime}\right|^{2}+\Omega^{2}\left|g_{\lambda}\right|^{2}}{2}, \quad F_{J}=\frac{\vartheta_{J}}{2}\left[g_{\lambda}^{\prime 2}+\Omega^{2} g_{\lambda}^{2}\right],
$$

and we have chosen such eigenfunctions $\Phi_{J}(\mathbf{x})$ that, for arbitrary $J$, there is such $\bar{J}$ that $\Phi_{J}^{*}(\mathbf{x})=$ $\vartheta_{J} \Phi_{\bar{J}}(\mathbf{x}),\left|\vartheta_{J}\right|=1,\left(\overline{\bar{J}}=J, \vartheta_{\bar{J}}=\vartheta_{J}\right)$. Such a choice is possible due to completeness and orthonormality of the set $\Phi_{J}(\mathbf{x})$.

In spherical coordinates of a homogeneous isotropic space, if $J=\{\lambda, l, \ldots, m\}$, we have $\bar{J}=$ $\{\lambda, l, \ldots,-m\}, \vartheta_{J}=(-1)^{m}$ (see [1]).

\section{HAMILTONIAN DIAGONALIZATION}

The Hamiltonian (15) will be diagonal at the time instant $\eta_{0}$ with respect to the operators $a_{J}^{( \pm)}, \stackrel{*}{a}{ }_{J}^{( \pm)}$, under the conditions (10). Diagonalization of the Hamiltonian at an arbitrary time instant $\eta$ is carried out in terms of the operators $b_{J}^{*}(\eta)$ and $b_{J}^{( \pm)}(\eta)$, connected with $\stackrel{*}{a} \underset{J}{( \pm)}, a_{J}^{( \pm)}$by time-dependent Bogoliubov transformations:

$$
\left\{\begin{array}{l}
a_{J}^{(-)}=\alpha_{J}^{*}(\eta) b_{J}^{(-)}(\eta)-\beta_{J}(\eta) \vartheta_{J} b_{\bar{J}}^{(+)}(\eta), \\
a_{J}^{*(-)}=\alpha_{J}^{*}(\eta) b_{J}^{(-)}(\eta)-\beta_{J}(\eta) \vartheta_{J} b_{\bar{J}}^{*(+)}(\eta),
\end{array}\right.
$$

where the functions $\alpha_{J}(\eta)=\alpha_{\bar{J}}(\eta)$ and $\beta_{J}(\eta)=$ $\beta_{\bar{J}}(\eta)$ satisfy the initial conditions $\left|\alpha_{J}\left(\eta_{0}\right)\right|=1$, $\beta_{J}\left(\eta_{0}\right)=0$ and the identity $\left|\alpha_{J}(\eta)\right|^{2}-\left|\beta_{J}(\eta)\right|^{2}=$ 1. Substituting (17) and the conjugate expressions to (15), one can obtain an expression for the Hamiltonian having the same form (15) but with the replacement $\stackrel{*}{a}( \pm), a_{J}^{( \pm)} \rightarrow \stackrel{*}{b}( \pm), b_{J}^{( \pm)}$and

$$
\begin{aligned}
& E_{J} \rightarrow{ }_{b} E_{J}=E_{J}\left(\left|\alpha_{J}\right|^{2}+\left|\beta_{J}\right|^{2}\right)-2 \operatorname{Re}\left(F_{J} \alpha_{J} \beta_{J}^{*} \vartheta_{J}^{*}\right), \\
& F_{J} \rightarrow{ }_{b} F_{J}=-2 \alpha_{J} \beta_{J} \vartheta_{J} E_{J}+\alpha_{J}^{2} F_{J}+\beta_{J}^{2} \vartheta_{J}^{2} F_{J}^{*} .
\end{aligned}
$$

From the diagonality requirement for the Hamiltonian with respect to the operators ${ }_{b J}^{*}( \pm)(\eta)$ and $b_{J}^{( \pm)}(\eta)$ at the time $\eta$, i.e., ${ }_{b} F_{J}(\eta)=0$, (for $\Omega^{2}(\eta)>$ 0 ), it follows:

$$
\alpha_{J}=i \chi_{J} \frac{g_{\lambda}^{* \prime}-i \Omega g_{\lambda}^{*}}{2 \sqrt{\Omega}}, \quad \beta_{J}=i \chi_{J} \frac{g_{\lambda}^{\prime}-i \Omega g_{\lambda}}{2 \sqrt{\Omega}},
$$

where $\chi_{J}=\chi_{\bar{J}}$ is an arbitrary complex function of time with a unit absolute value. Therefore further we will use the operators

$$
c_{J}^{(+)}(\eta)=\chi_{J}(\eta) b_{J}^{(+)}(\eta), \quad c_{J}^{(-)}(\eta)=\chi_{J}^{*}(\eta) b_{J}^{(-)}(\eta),
$$

which, due to (17) and (20), do not depend on the specific choice of the functions $\chi_{J}(\eta)$. The operators ${ }_{c_{J}^{*}}^{*( \pm)}(\eta)$ and $c_{J}^{( \pm)}(\eta)$ obey the same commutation relations as $\stackrel{*}{a}_{J}^{( \pm)}$and $a_{J}^{( \pm)}$.

Substitution of (21), taking into account (17) and their conjugate expressions, into (15) leads to the following expression for the Hamiltonian:

$$
H(\eta)=\int d \mu(J) \Omega(\eta)\left(\stackrel{*}{c}_{J}^{(+)} c_{J}^{(-)}+\stackrel{*}{c}_{J}^{(-)} c_{J}^{(+)}\right) .
$$

Thus the energy of quasiparticles corresponding to the diagonal form of the Hamiltonian (15) is equal to the oscillator frequency $\Omega(\eta)$ (unlike the Hamiltonian built from the metric stress-energy tensor of a nonconformal scalar field 6]).

Using (7), (17), (20), and (22), one can verify that the operators $c_{J}^{( \pm)}(\eta)$ obey Heisenberg's equations of motion:

$$
\frac{d c_{J}^{( \pm)}}{d \eta}=i\left[H(\eta), c_{J}^{( \pm)}\right]+\frac{\Omega^{\prime}}{2 \Omega} \vartheta_{J}^{(\mp 1)} c_{\bar{J}}^{(\mp)} .
$$


The second term in the right-hand side of (23) is connected with re-definition of the particle notion at each time instant.

An expansion of the field operator $\tilde{\varphi}(x)$ in the operators $c_{J}^{( \pm)}(\eta)$ :

$$
\tilde{\varphi}(x)=\int \frac{d \mu(J)}{\sqrt{2 \Omega}}\left[\Phi_{J}^{*}(\mathbf{x}) c_{J}^{(+)}(\eta)+\Phi_{\bar{J}}(\mathbf{x}) c_{\bar{J}}^{(-)}(\eta)\right]
$$

follows from (11), (12), (17), and (20). The equations of motion hold for each mode in (24) separately.

Consider the question of particle creation in a nonstationary metric. We suppose that the quantized scalar field is in the state $|0\rangle$, annihilated by the operators $a_{J}^{(-)}, \stackrel{*}{a}_{J}^{(-)}$, i.e., in the vacuum state for the instant $\eta_{0}$. At the time $\eta$, the vacuum state is the state $\left|0_{\eta}\right\rangle$, defined by the equalities

$$
c_{J}^{(-)}(\eta)\left|0_{\eta}\right\rangle=\stackrel{*}{c}_{J}^{(-)}(\eta)\left|0_{\eta}\right\rangle=0 .
$$

The state $|0\rangle$ contains, at the time instant $\eta$, $\left|\beta_{J}(\eta)\right|^{2}$ pairs of particles and antiparticles corresponding to the operators $c_{J}^{( \pm)}(\eta)$ (see [1]). The density of the created particle pairs may be calculated (for the quasi-Euclidean metric with $K=0$ ) by the formula

$$
n(\eta)=\frac{B_{N}}{2 a^{N-1}} \int_{0}^{\infty} S_{\lambda}(\eta) \lambda^{N-2} d \lambda,
$$

where $B_{N}=\left[2^{N-3} \pi^{(N-1) / 2} \Gamma((N-1) / 2)\right]^{-1}, \Gamma(z)$ is the gamma function, $S_{\lambda}(\eta)=\left|\beta_{\lambda}(\eta)\right|^{2}$ (in a homogeneous isotropic space, $\left.\left|\beta_{J}\right| \equiv\left|\beta_{\lambda}\right|\right)$. For $N=4$ and $K=0,-1$, for the number density of the particle pairs created, the following formula is valid (see [1]):

$$
n(\eta)=\frac{1}{2 \pi^{2} a^{3}} \int_{0}^{\infty} S_{\lambda}(\eta) \lambda^{2} d \lambda,
$$

For $K=1$ (spherical space), the set of eigenfunctions of the Laplace-Beltrami operator $\Delta_{N-1}$ is discrete, and the formula for the number density of the created particle pairs $N=4$ has the form (see [1])

$$
n(\eta)=\frac{1}{2 \pi^{2} a^{3}} \sum_{\lambda=1}^{\infty} S_{\lambda}(\eta) \lambda^{2} .
$$

Using (20) and that the function

$$
g_{\lambda}(\eta) g_{\lambda}^{* \prime}(\eta)-g_{\lambda}^{\prime}(\eta) g_{\lambda}^{*}(\eta)
$$

is a first integral of the equation (7), equal to $-2 i$ according to the initial conditions (10), we obtain:

$$
S_{\lambda}(\eta)=\frac{1}{4 \Omega}\left(\left|g_{\lambda}^{\prime}\right|^{2}+\Omega^{2}\left|g_{\lambda}\right|^{2}\right)-\frac{1}{2} .
$$

As it was shown in [10], $S_{\lambda}(\eta) \sim \lambda^{-6}$ as $\lambda \rightarrow$ $\infty$. Therefore, in four-dimensional space-time, the number density of particles created, defined by the Hamiltonian (14) diagonalization method, is finite in the nonconformal case as well. Let us note that a divergent expression for the number density of created nonconformal scalar particles, obtained with another choice of the Hamiltonian in Ref. 11, has been one of the reasons for a criticism of the Hamiltonian diagonalization method as a whole in the well-known book 2].

\section{THE SPACE-TIME CORRELATION FUNCTION}

To study the space-time characteristics of the created quasiparticles, we apply the approach suggested in [5. We use the notion of a particle's localized state introduced by Newton and Wigner [15]. By analogy with the Newton-Wigner operator for a free field (see, e.g., [16]), we introduce creation operators of a localized state of a particle and an antiparticle:

$$
\begin{aligned}
& \stackrel{*}{\varphi}_{1}^{(+)}(\eta, \mathbf{x})=a^{-\frac{N-1}{2}}(\eta) \int d \mu(J) \Phi_{J}^{*}(\mathbf{x}) \stackrel{*}{c}(+)_{J}^{(+)}(\eta), \\
& \varphi_{1}^{(+)}(\eta, \mathbf{x})=a^{-\frac{N-1}{2}}(\eta) \int d \mu(J) \Phi_{J}^{*}(\mathbf{x}) c_{J}^{(+)}(\eta)
\end{aligned}
$$

By analogy with the case of a conformal scalar field considered in [1], we introduce the operator of particle number in the volume $V$

$$
N_{V}=\int_{V} d^{N-1} x \sqrt{{ }^{(N-1)} g} \stackrel{*}{\varphi}_{1}^{(+)}(\eta, \mathbf{x}) \varphi_{1}^{(-)}(\eta, \mathbf{x}),
$$

$$
\begin{array}{r}
\text { where }{ }^{(N-1)} g=\operatorname{det}\left({ }^{(N-1)} g_{\alpha \beta}\right), \text { and } \\
{ }^{(N-1)} g_{\alpha \beta}=a^{2}(\eta) \gamma_{\alpha \beta}
\end{array}
$$

is the induce metric tensor on the hypersurface $\eta=$ const. Using (17), (21), (30) and the properties of the eigenfunctions $\Phi_{J}(\mathbf{x})$ (see, e.g., 1], $\S 9.1$ ), it can be shown that the expression for the number density of the particles created

$$
n=\left\langle 0\left|N_{V}\right| 0\right\rangle / V
$$

obtained with the aid of (31), reproduces Eqs. (26)(28).

As in [5], we consider as a characteristic of the spatial distribution of the quasiparticle pairs created, the matrix element

$$
R_{0}\left(\eta, \mathbf{x}, \mathbf{x}^{\prime}\right)=\frac{\left\langle 0_{\eta}\left|\varphi_{1}^{(-)}(\eta, \mathbf{x}) \stackrel{*}{\varphi}_{1}^{(-)}\left(\eta, \mathbf{x}^{\prime}\right)\right| 0\right\rangle}{\left\langle 0_{\eta} \mid 0\right\rangle}
$$


which has the meaning of the probability amplitude that a quasiparticle created is located at the point $\mathbf{x}$ at the time instant $\eta$ while the antiquasiparticle is at the point $\mathbf{x}^{\prime}$. Using (17), (20), (21) and (30), we obtain:

$$
R_{0}\left(\eta, \mathbf{x}, \mathbf{x}^{\prime}\right)=\frac{1}{a^{N-1}(\eta)} \int d \mu(J) \Phi_{J}(\mathbf{x}) \Phi_{J}^{*}\left(\mathbf{x}^{\prime}\right) P_{\lambda}(\eta),
$$

where

$$
P_{\lambda}(\eta)=\left(i \Omega g_{\lambda}-g_{\lambda}^{\prime}\right) /\left(i \Omega g_{\lambda}+g_{\lambda}^{\prime}\right) .
$$

The function $P_{\lambda}(\eta)$ satisfies the following equation and initial condition:

$$
P_{\lambda}^{\prime}+2 i \Omega P_{\lambda}+\frac{\Omega^{\prime}}{2 \Omega}\left(P_{\lambda}^{2}-1\right)=0, \quad P_{\lambda}\left(\eta_{0}\right)=0 .
$$

Consider the case that the metric is changing adiabatically:

$$
\frac{1}{\Omega}\left|\left(\frac{\Omega^{\prime}}{\Omega^{2}}\right)^{\prime}\right| \ll\left|\frac{\Omega^{\prime}}{\Omega^{2}}\right| \ll 1 .
$$

Furthermore, we denote $M=\sqrt{\Omega^{2}-\lambda^{2}} / a$. In the general case, $M=M(\eta)$. For a conformally coupled scalar field, $M=m$. If $M=$ const, the conditions (37) hold if $\dot{h}(t) / M^{2} \ll h / M \ll 1$, where $h(t)=\dot{a}(t) / a$ is the Hubble parameter.

If the conditions (37) and $a^{\prime}\left(\eta_{0}\right)=0$ are satisfied, an approximate solution to (36) has the form

$$
P_{J}(\eta) \approx-i \Omega^{\prime} /\left(4 \Omega^{2}\right) .
$$

Let us find an expression for the space-time correlation function (33) in the approximation considered in the quasi-Euclidean metric (i.e., $d s^{2}=$ $\left.a^{2}(\eta)\left(d \eta^{2}-d x^{\alpha} d x^{\alpha}\right)\right)$. The eigenfunctions of the Laplace operator $\Delta_{N-1}$ in the coordinates $x^{\alpha}$ are

$$
\Phi_{J}(\mathbf{x})=(2 \pi)^{(1-N) / 2} \exp \left(-i \lambda_{\alpha} x^{\alpha}\right),
$$

where $-\infty<\lambda_{a}<+\infty, \quad \sum_{\alpha} \lambda_{\alpha}^{2}=\lambda^{2}$. Consequently,

$$
\sum_{J(\lambda=\text { const })} \Phi_{J}^{*}(\mathbf{x}) \Phi_{J}\left(\mathbf{x}^{\prime}\right)=\left(\frac{\lambda}{2 \pi}\right)^{\frac{N-1}{2}} \frac{J_{(N-3) / 2}(\lambda \rho)}{\rho^{(N-3) / 2}},
$$

where $\sum=\int d \vec{\lambda} \delta(|\vec{\lambda}|-\lambda), \quad \rho=\left|\mathbf{x}-\mathbf{x}^{\prime}\right|$, and $J_{\nu}(z)$ are Bessel functions. Substituting (38), (40) into (34) and performing integration, we obtain

$$
R_{0}\left(\eta, \mathbf{x}, \mathbf{x}^{\prime}\right)=\frac{-i(M a)^{2 \prime}}{16 \pi^{2} a^{3}}\left(\frac{M}{2 \pi r}\right)^{\frac{N-4}{2}} K_{\frac{N-4}{2}}(M r),
$$

where $r=\rho a$ and $K_{\nu}(z)$ are MacDonald's functions.
If $M=$ const, which is the case for a conformally coupled scalar field and for an arbitrary coupled field in de Sitter space, from (41) we obtain

$$
R_{0}\left(t, \mathbf{x}, \mathbf{x}^{\prime}\right)=\frac{-i M^{2} h(t)}{8 \pi^{2}}\left(\frac{M}{2 \pi r}\right)^{\frac{N-4}{2}} K_{\frac{N-4}{2}}(M r) .
$$

Let us further consider spatial sections with $K=$ \pm 1 . For $N=4$, the space-time metric may be written in the form

$$
d l^{2}=d \chi^{2}+f^{2}(\chi)\left(d \vartheta^{2}+\sin ^{2} \vartheta d \varphi^{2}\right),
$$

where $f(\chi)=\sinh (\chi), \chi, \sin (\chi)$ for $K=-1,0,+1$, respectively. Meanwhile,

$$
\sum_{J(\lambda=\text { const })} \Phi_{J}^{*}(\mathbf{x}) \Phi_{J}\left(\mathbf{x}^{\prime}\right)=\frac{\lambda}{2 \pi^{2}} \frac{\sin \lambda \rho}{f(\rho)},
$$

where $\rho$ is the geodesic distance between the points $\mathbf{x}$ and $\mathbf{x}^{\prime}$ (see [1). Therefore, from (34) and (38) we obtain in the hyperbolic $(K=-1)$ case:

$$
R_{0}\left(\eta, \mathbf{x}, \mathbf{x}^{\prime}\right)=\frac{-i(M a)^{2 \prime}}{16 \pi^{2} a^{3}} \frac{\rho}{\sinh \rho} K_{0}(M r) .
$$

If $|z| \gg 1$, then $K_{\nu}(z) \sim \sqrt{\pi /(2 z)} e^{-z}$. Consequently, if the metric is changing adiabatically, the function $\left|R_{0}\right|$ in the quasi-Euclidean (see (410) and hyperbolic space-time decreases exponentially with growing $\rho$ at spacings $r \gg 1 / M$, i.e., exceeding the Compton wavelength.

In spherical case, the spacing $\rho$ changes in the range $0 \leq \rho \leq \pi$. A substitution of (38) and (44) into (34) leads to

$$
R_{0}\left(\eta, \mathbf{x}, \mathbf{x}^{\prime}\right)=\frac{-i(M a)^{2 \prime}}{16 \pi^{2} a^{3} \sin \rho} \sum_{\lambda=1}^{\infty} \frac{\lambda \sin \lambda \rho}{\left(M^{2} a^{2}+\lambda^{2}\right)^{3 / 2}} .
$$

The sum in the right-hand side of (46), after a chain of transformations, may be presented in the form

$$
\begin{aligned}
& \sum_{n=0}^{\infty}(\rho+2 \pi n) K_{0}(M a(\rho+2 \pi n))- \\
& -\sum_{n=1}^{\infty}(2 \pi n-\rho) K_{0}(M a(2 \pi n-\rho)) .
\end{aligned}
$$

From the asymptotic properties of the function $K_{0}(z)$ it follows that, for $\rho \ll 1$ and $M a \gg 1$ (the distance between particles od a pair and a particle's Compton wavelength are much smaller than the curvature radius of space), in this representation one could retain only the term $\rho K_{0}(M a \rho)$, and therefore Eq. (46) takes the form

$$
R_{0}\left(\eta, \mathbf{x}, \mathbf{x}^{\prime}\right)=\frac{-i(M a)^{2 \prime}}{16 \pi^{2} a^{3}} \frac{\rho}{\sin \rho} K_{0}(M r) .
$$


Therefore, in the spherical case $\left|R_{0}\right|$ also decreases exponentially with growing $\rho$ at distances $r \gg 1 / M$, exceeding the Compton wavelength.

Thus if the metric of a homogeneous isotropic state is changing adiabatically, the space-time correlation function $R_{0}\left(\eta, \mathbf{x}, \mathbf{x}^{\prime}\right)$ is exponentially small for $r \gg 1 / M$. This indicates that the corresponding quasiparticles are virtual pairs with the characteristic correlation length equal to $1 / M$. Real particle creation is exponentially small and does not manifest itself in perturbation theory.

\section{PARTICLE CREATION IN DE SITTER SPACE}

Let us consider de Sitter space and take the metric in the form (5) with $K=0$ and

$$
a=a_{1} e^{H t}=-\frac{1}{H \eta},
$$

$t \in(-\infty,+\infty) \Leftrightarrow \eta \in(-\infty, 0)$.

Solutions to Eq. (7) with $V_{g}=\xi R$ and the conditions (10) for $\eta_{0} \rightarrow-\infty$ have the form

$$
g_{\lambda}(\eta)=\sqrt{-\frac{\pi \eta}{2}} e^{\frac{\pi}{2} \operatorname{Im} \nu} H_{\nu}^{(2)}(-\lambda \eta) e^{i \alpha_{0}}
$$

where $H_{\nu}^{(2)}(z)$ is a Hankel function, $\alpha_{0}$ is an arbitrary real constant,

$$
\nu=\sqrt{\frac{1}{4}-\left(\frac{M}{H}\right)^{2}}, \quad M=\sqrt{m^{2}+\left(\xi-\xi_{c}\right) R},
$$

and $R=N(N-1) H^{2}$.

Furthermore, assuming $m^{2}+\left(\xi-\xi_{c}\right) R>0$, from (26), (29) and (50) we obtain

$$
n=M^{N-1} \cdot F_{N}\left(\frac{M}{H}\right)
$$

i.e., the created particle number density in de Sitter space is time-independent! The result of a numerical computation for the function $F_{N}(M / H)$ in the case $N=4$ is represented in Fig. 1 .

At $M / H \gg 1$, the metric is changing adiabatically and, as shown in Section 4, real particle creation does not occur.

In the general case, substituting the exact solution (50) into (34) and (35) and using (40), we obtain

$$
\frac{R_{0}\left(t, \mathbf{x}, \mathbf{x}^{\prime}\right)}{M^{N-1}}=f\left(M r, \frac{M}{H}\right),
$$

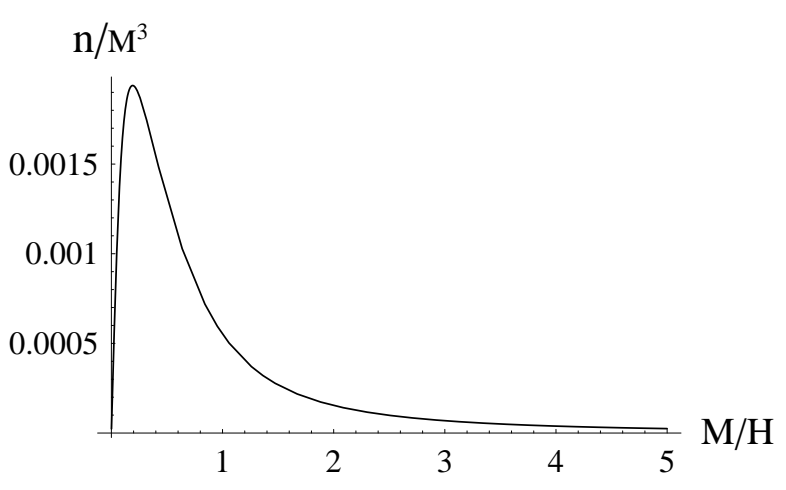

Figure 1: The number density of quasiparticles created in de Sitter space.

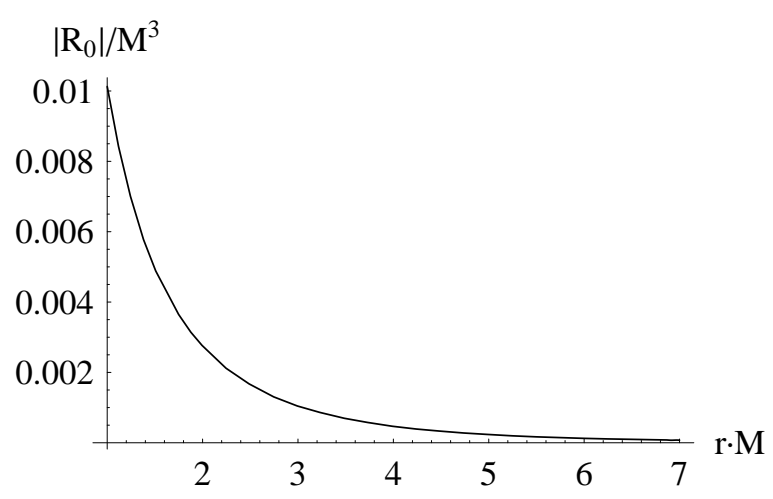

Figure 2: The correlation function for $M / H=0.01$.

where $r=a(t)\left|\mathbf{x}^{\prime}-\mathbf{x}\right|$, i.e., the correlation function, expressed in terms of $r$ (the "physical" distance between the quasiparticles in a pair) is timeindependent.

Examples of numerical calculations for $N=4$, $M / H=0.01$ and $M / H=0.2$ are given in Figs. 2 and 3, respectively.

In both cases, the correlation function decreases exponentially at distances between the quasiparticles exceeding the Compton wavelength $l_{C}=1 / M$.

Thus real particles creation in de Sitter space does not occur. The quasiparticle pairs being created, whose density has been calculated and shown in Fig. 1, should be interpreted as pairs of virtual particles.

As has been noticed in [1, the absence of real particle creation in de Sitter space is confirmed by the local nature of the vacuum stress-energy tensor and by vanishing of the imaginary part of the effective Lagrangian. 


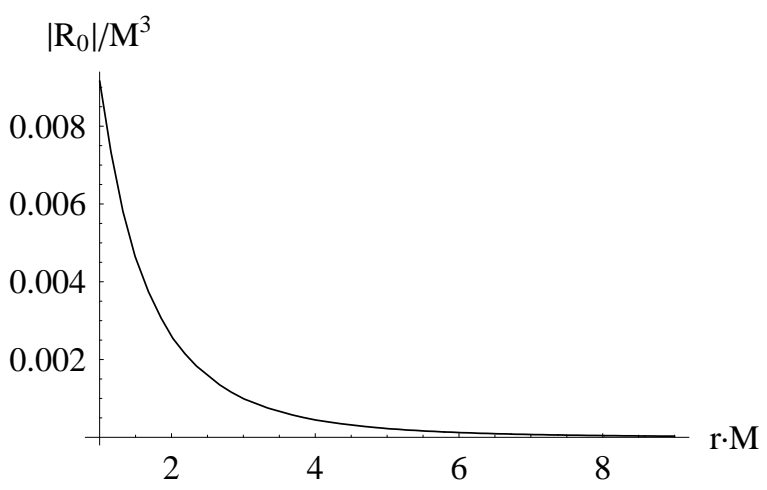

Figure 3: The correlation function for $M / H=0.2$.

\section{CONCLUSIONS}

In this paper, for a scalar field nonconformally coupled to the curvature, we gave a generalization of the method of space-time description of particle creation by the gravitational field. In a homogeneous isotropic space, we have introduced the creation operators (30) of localized one-particle states and the operator (31) of particle number in a specified volume. We have obtained the expressions (34) and (35) for the space-time correlation function (33) of a pair of created quasiparticles corresponding to a diagonal form of the instantaneous Hamiltonian. We have analyzed the case of adiabatic changes in the metric of a homogeneous isotropic space. The expressions (41), (42), (45) and (46) have been obtained for the correlation function of a pair of quasiparticles created. It has been shown that the correlation function exponentially decreases at spaces exceeding the Compton wavelength, and consequently real particle creation is suppressed. Particle creation in de Sitter space has been considered, and, from, the properties of the space-time correlation function for a pair of quasiparticles created, it has been concluded that such a pair should be interpreted as a pair of virtual particles.

\section{ACKNOWLEDGMENTS}

The author thanks Prof. A.A. Grib for helpful discussions. The work has been financially supported by RNP Grant 2.1.1.6826.

\section{REFERENCES}

[1] A. A. Grib, S. G. Mamayev, and V. M. Mostepanenko, Vacuum Quantum Effects in Strong
Fields (Energoatomizdat, Moscow, 1988, in Russian; English translation: Friedmann Lab. Publ., St.Petersburg, 1994).

[2] N.D. Birrell and P.C.W. Davies, Quantum Fields in Curved Space (Cambridge Univ. Press, Cambridge, 1982).

[3] A. A. Grib and Yu. V. Pavlov, Int. J. Mod. Phys. D 11, 433 (2002); Int. J. Mod. Phys. A 17, 4435 (2002); Grav. \& Cosmol. 12, 159 (2006); Grav. \& Cosmol. 14, 1 (2008).

[4] A. A. Grib and S. G. Mamayev, Yadernaya Fizika 10, 1276 (1969); Sov. J. Nucl. Phys. 10, 722 (1970).

[5] S. G. Mamayev and N. N. Trunov, Yadernaya Fizika 37, 1603 (1983); Sov. J. Nucl. Phys. 37, 952 (1983).

[6] V. B. Bezerra, V. M. Mostepanenko, and C. Romero, Int. J. Mod. Phys. D 7, 249 (1998).

[7] M. Bordag, J. Lindig, V. M. Mostepanenko, and Yu. V. Pavlov, Int. J. Mod. Phys. D 6, 449 (1997).

[8] L.P. Grishchuk and V.M. Yudin, J. Math. Phys. 21, 1168 (1980).

[9] X.S. Mamaeva and N. N. Trunov, Teor. Mat. Fiz. 135, 82 (2003); Theor. Math. Phys. 135, 520 (2003).

[10] Yu. V. Pavlov, Teor. Mat. Fiz. 126, 115 (2001); Theor. Math. Phys. 126, 92 (2001).

[11] S. A. Fulling, Gen. Relativ. Gravit. 10, 807 (1979).

[12] Yu. V. Pavlov, Teor. Mat. Fiz. 140, 241 (2004); Theor. Math. Phys. 140, 1095 (2004).

[13] C. Lanczos, Ann. Math. 39, 842 (1938).

[14] Yu. V. Pavlov, Int. J. Mod. Phys. A 17, 1041 (2002).

[15] T.D. Newton and E.P. Wigner, Rev. Mod. Phys. 21, 400 (1949).

[16] S. S. Schweber, An Introduction to Relativistic Quantum Field Theory (Row, Peterson and Co, New York, 1961). 